\title{
Decision Making in Interventional Oncology: Ablative Options in the Lung
}

\author{
Kyungmouk Steve Lee, MD ${ }^{1}$ Joseph P. Erinjeri, MD, $\mathrm{PhD}^{2}$ \\ ${ }^{1}$ Department of Radiology, Weill Cornell Medicine, New York \\ ${ }^{2}$ Department of Radiology, Memorial Sloan-Kettering Cancer Center, \\ New York \\ Address for correspondence Joseph P. Erinjeri, MD, PhD, \\ Interventional Radiology Service, Memorial Sloan Kettering Cancer \\ Center, 1275 York Avenue - H118, New York, NY 10065 \\ (e-mail: erinjerj@mskcc.org). \\ Semin Intervent Radiol 2017;34:176-181.
}
Abstract
Keywords
- lung ablation
- image-guided ablation
- lung cancer
- interventional radiology

\begin{abstract}
Image-guided ablation is safe and effective for the treatment of both primary and metastatic tumors in the lung. This article reviews the three most commonly used ablative options: radiofrequency ablation, microwave ablation, and cryoablation. We describe the advantages of each ablation modality in the lung and how to choose the most appropriate ablation device based on patient and tumor characteristics. The optimal technique for lung ablation is discussed and technical tips for improving clinical outcome are described.
\end{abstract}

Objectives: Upon completion of this article, the reader will be able to identify the ablative options in the lung and explain the decision-making process for choosing an appropriate ablation modality for each individual patient.

Accreditation: This activity has been planned and implemented in accordance with the Essential Areas and Policies of the Accreditation Council for Continuing Medical Education (ACCME) through the joint providership of Tufts University School of Medicine (TUSM) and Thieme Medical Publishers, New York. TUSM is accredited by the ACCME to provide continuing medical education for physicians.

Credit: Tufts University School of Medicine designates this journal-based CME activity for a maximum of 1 AMA PRA Category 1 Credit $^{\mathrm{TM}}$. Physicians should claim only the credit commensurate with the extent of their participation in the activity.

Lung cancer is the leading cause of cancer death in both the United States and in the world. ${ }^{1,2}$ The 5-year survival rate for lung cancer is $17.4 \%$, and more than half of lung cancer patients die within 1 year of diagnosis. ${ }^{2}$ Surgery is the standard-of-care treatment for patients with early-stage lung cancer. However, a majority of patients with lung cancer are not surgical candidates or medically inoperable due to comorbidities. In these patients, image-guided ablation may offer an alternative treatment option. The National Comprehensive Cancer Network guidelines include image-guided ablation as a treatment option for selected, medically inoperable patients with stage IA NSCLC. Ablation is also considered a treatment strategy for patients with multiple lung cancers and locoregional tumor recurrence. Compared with surgery, the advantages of ablation include minimal invasiveness with lesser impact on pulmonary function as well as cost-effectiveness, as it can often be performed on an outpatient basis. ${ }^{3,4}$ Ablation can also be performed repeatedly without dose limitations and may be useful for lung tumors resistant to radiation therapy (e.g., colorectal metastases). ${ }^{5}$ Currently, there are no randomized clinical trials comparing image-guided ablation with radiation therapy or surgery.

Since the first reported use of radiofrequency ablation (RFA) in the lung by Dupuy et al in $2000,{ }^{6}$ various imageguided ablative techniques have been used to treat pulmonary tumors. Although RFA has historically been the most commonly used device, microwave ablation (MWA) and cryoablation are now favored modalities for lung ablation. In the lung, high-powered MWA generates larger ablation zones more quickly and more predictably compared with RFA. Cryoablation is generally preferred over RFA in the lung for larger peripheral tumors invading the chest wall or central tumors involving the mediastinum. Although there have not been clinical trials directly comparing the various ablative techniques, patient and tumor characteristics are used to determine which ablation device should be used. In this article, we review the three most commonly used ablation options in the lung (RFA, MWA, and cryoablation).
Issue Theme Decision Making as a Growth Mechanism in Interventional Oncology; Guest Editor, Daniel B. Brown, MD, FSIR
Copyright (c) 2017 by Thieme Medical Publishers, Inc., 333 Seventh Avenue, New York, NY 10001, USA. Tel: +1(212) 584-4662. 


\section{Ablative Techniques}

\section{Radiofrequency Ablation}

In RFA, an electrode is placed into the tumor under CT imaging guidance (-Fig. 1). The electrode is connected to an RF generator, and a voltage is produced between the electrode and the grounding pad. As this voltage or electric field oscillates (typically from 375 to $500 \mathrm{kHz}$ ), frictional heat is produced and results in coagulation necrosis and cell death at temperatures greater than $60^{\circ} \mathrm{C}$. The goal is to create an ablation zone large enough to encompass the entire tumor with a surrounding margin. Several RFA systems are commercially available. Two of the RFA systems use electrodes with 4 to 16 small wires or tines that are deployed via a needle (14-17 gauge). ${ }^{7,8}$ Another RFA system employs a single or triple cluster of electrodes that are spaced $5 \mathrm{~mm}$ apart with the tip seeded in the tumor. ${ }^{9}$

The majority of the safety and efficacy data on lung ablation come from RFA. RFA has been shown to be tolerated well by patients with both primary NSCLC and pulmonary metastases. ${ }^{10-14}$ Better outcomes are achieved with smaller tumors (typically $<3 \mathrm{~cm}$ in diameter for RFA). In the American College of Surgeons Oncology Group Z4033 trial, RFA was used to treat medically inoperable patients with stage IA NSCLC. The overall survival was $86.3 \%$ at 1 year and $69.8 \%$ at 2 years with a local recurrence-free rate of $68.9 \%$ at 1 year and $59.8 \%$ at 2 years. ${ }^{15}$ Improvement in survival was noted in patients with tumors smaller than $2.0 \mathrm{~cm}$ and performance status of 0 or 1 . No grade 4 or 5 adverse events were noted and there was also no significant change in pulmonary function.

In the RAPTURE study (a prospective, intention-to-treat, multicenter trial), RFA was used to treat 106 patients with 183 lung tumors smaller than $3.5 \mathrm{~cm}$ (33 patients with primary NSCLC, 73 patients with pulmonary metastases). ${ }^{16}$ The 1-year complete response rate was $88 \%$ overall, while the 2-year cancer-specific survival was $92 \%$ for primary NSCLC. In a study of 566 patients with 1,037 pulmonary metastases, RFA was an effective treatment option for nodules smaller than 2 to $3 \mathrm{~cm}$ with a 4 -year local efficacy of $89 \%$; in this large study, the most common primary sites were colorectal (52\%), renal (18\%), and sarcoma $(9 \%){ }^{14}$

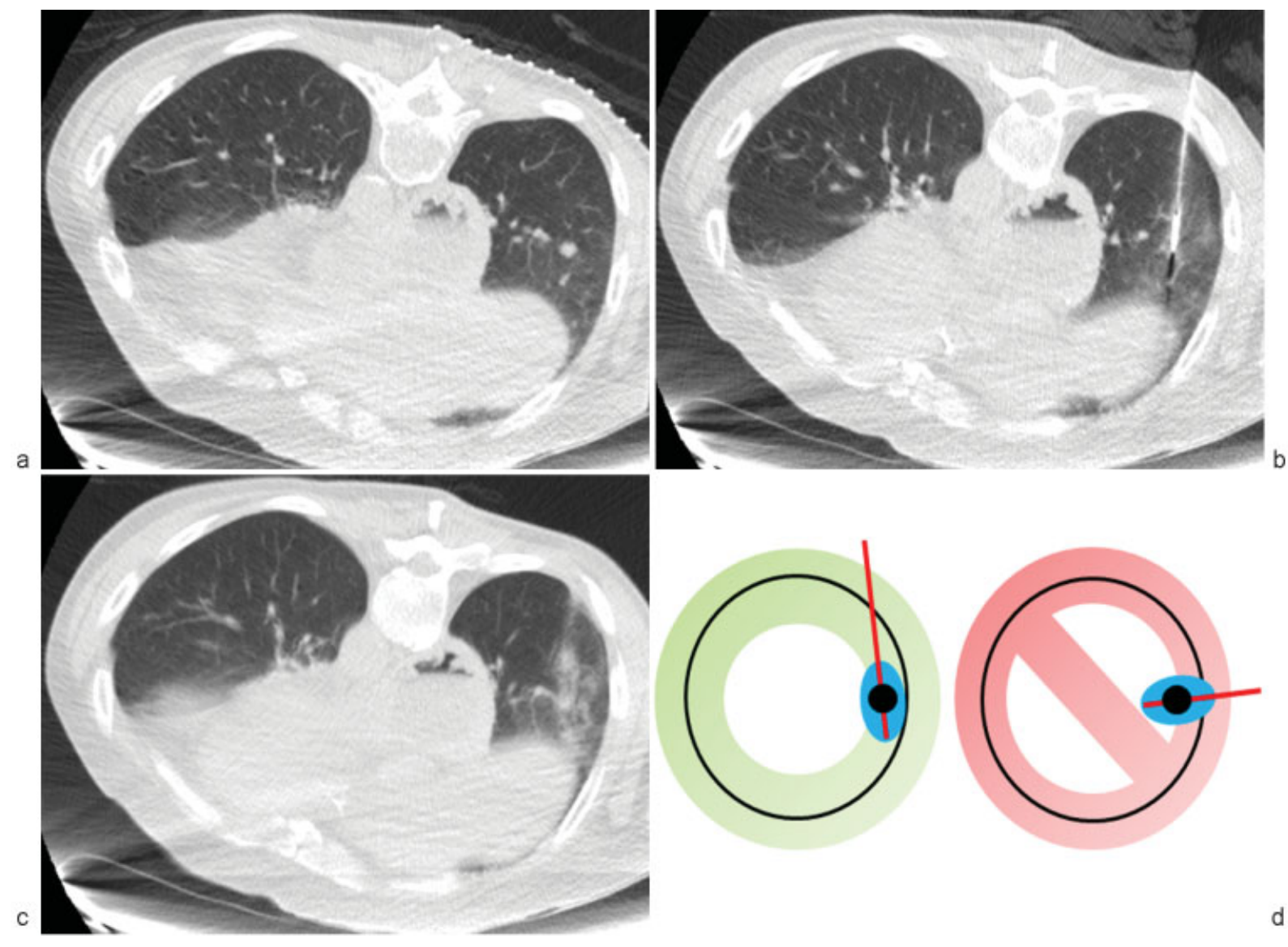

Fig. 1 Microwave ablation of a lung nodule in a patient who is not a surgical candidate. (a) Preprocedural CT image shows a nodule in the right lower lobe (arrow-away from the chest wall and mediastinum) that is amenable for microwave ablation. (b) Intraprocedural CT image shows the microwave ablation antenna (arrow) traversing lung parenchyma with distal end advanced into the nodule. (c) Postprocedural CT image shows the microwave ablation zone encompassing the nodule. Tract cautery should not be performed, as it may lead to prolonged air leaks and bronchopleural fistulae. (d) Schematic diagram showing recommended ablation probe trajectory for a peripheral lung nodule (left): the point of entry into lung (i.e., pleural puncture site) excludes the target ablation zone. It may be tempting to perform the ablation using the shortest, simplest path (right), but a "direct" path to the tumor may extend the ablation zone unnecessarily into the chest wall (red bar = ablation probe; blue oval = ablation zone; black dot = lung nodule; black circle $=$ pleural surface). 


\section{Microwave Ablation}

Microwave ablation uses an antenna to deposit focused energy directly into the lung for tumor destruction. Compared with RFA, MWA uses electromagnetic energy at a much higher frequency range (300 $\mathrm{MHz}$ to $300 \mathrm{GHz}$ ). This oscillating microwave field results in the induction of kinetic energy in polar water molecules, which in turn causes tissue heating and tumor destruction. Microwave energy can even penetrate tissues with high electric impedance such as the lung. Therefore, MWA can provide larger ablative zones that are less susceptible to heat sink effect. ${ }^{17}$ Multiple MWA systems are commercially available including devices with a straight 14- to 17-gauge antenna (with active tip length of $0.6-4.0 \mathrm{~cm}$ ) connected to a $915-\mathrm{MHz}$ generator $^{18-20}$ or a 2450-MHz generator. ${ }^{21-23}$

In a retrospective study of 50 patients, MWA was used to treat 82 pulmonary tumors (mean size, $3.5 \mathrm{~cm}$ ). The cancerspecific mortality analysis yielded a 1 -year survival of $83 \%$, a 2 -year survival of $73 \%$, and a 3 -year survival of $61 \% .{ }^{24}$ In this MWA study, imaging finding of cavitation in the ablated tumor had an association with lower cancer-specific mortality. Other MWA studies have shown that local tumor control is inversely related to tumor size. ${ }^{25,26}$

\section{Cryoablation}

In contrast to heat-based thermal ablation modalities (MWA and RFA), cryoablation uses pressurized argon gas to generate extremely cold temperatures of less than $-40^{\circ} \mathrm{C}$ to cause tumor necrosis. The mechanisms of cryogenic tissue destruction include cell rupture, protein denaturation, and microvascular thrombosis-induced ischemia. ${ }^{27}$ Cryoablation is performed using freeze-thaw-freeze cycles that cause thermal coagulation. ${ }^{28}$ During the thaw phase of the cycle, helium is used to increase the temperature of the cryoablation probe to approximately $20^{\circ} \mathrm{C}$. One advantage of cryoablation over heat-based ablation devices is the visualization of the ablation zone on $\mathrm{CT}$ imaging. Accurate visualization of ablation margin is critical to a successful cryoablation. The reason is that the cytotoxic margin of a cryoablation zone can be up to $7 \mathrm{~mm}$ away from the most periphery of the visualized "ice ball" on CT imaging. Therefore, the ice ball must be large enough to cover the entire tumor with at least 7-mm margin all around the tumor. ${ }^{29}$ Another benefit of cryoablation is its analgesic effect due to its mechanism of action; for example, lung cryoablation has been performed with local anesthesia only. ${ }^{30}$ Most importantly, cryoablation is generally considered safer than RFA or MWA for tumors near the chest wall or mediastinum (-Figs. 2 and 3). ${ }^{31}$ In the past, the major disadvantage of cryoablation was related to its lack of cautery effect resulting in potential increase in bleeding complications: pulmonary hemorrhage, hemoptysis, or hemothorax. However, newer cryoablation devices have a cautery option that may be used. The commercially available percutaneous
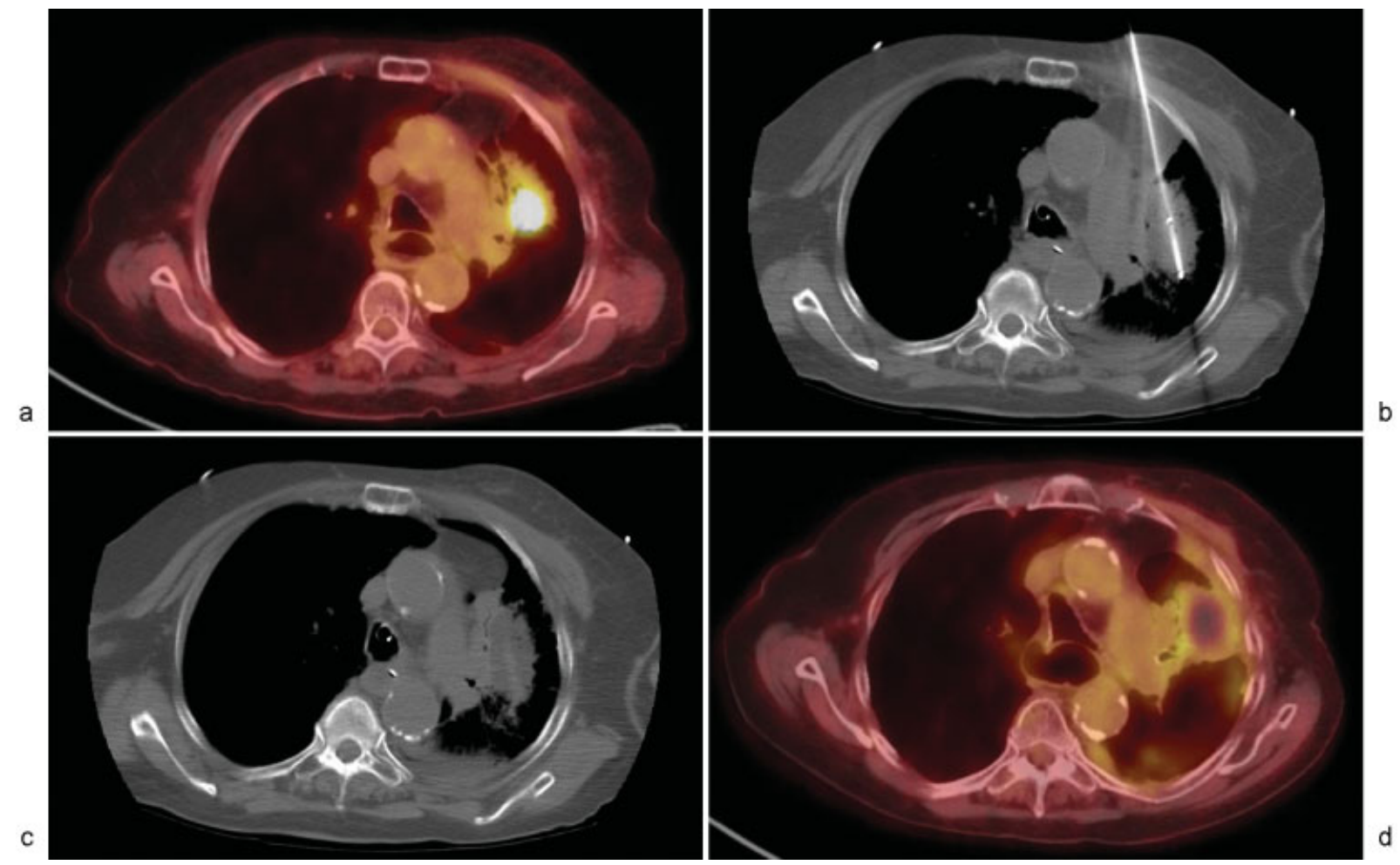

Fig. 2 Cryoablation of lung tumor recurrence resistant to radiation therapy. Cryoablation was chosen over RFA or MWA because of its higher safety profile for tumors in or near the mediastinum. However, in patients who have received radiation therapy, ablation should be used with caution as there is poor wound healing. (a) Preprocedural PET/CT image shows an FDG-avid mass near the mediastinum in the left lung. (b) Intraprocedural CT image shows cryoablation probe in the center of the tumor. (c) Immediate postprocedural CT image shows the hypodense "ice ball" encompassing the pulmonary tumor (arrow). (d) Postprocedural PET/CT image shows resolution of hypermetabolic activity in the left lung tumor. 


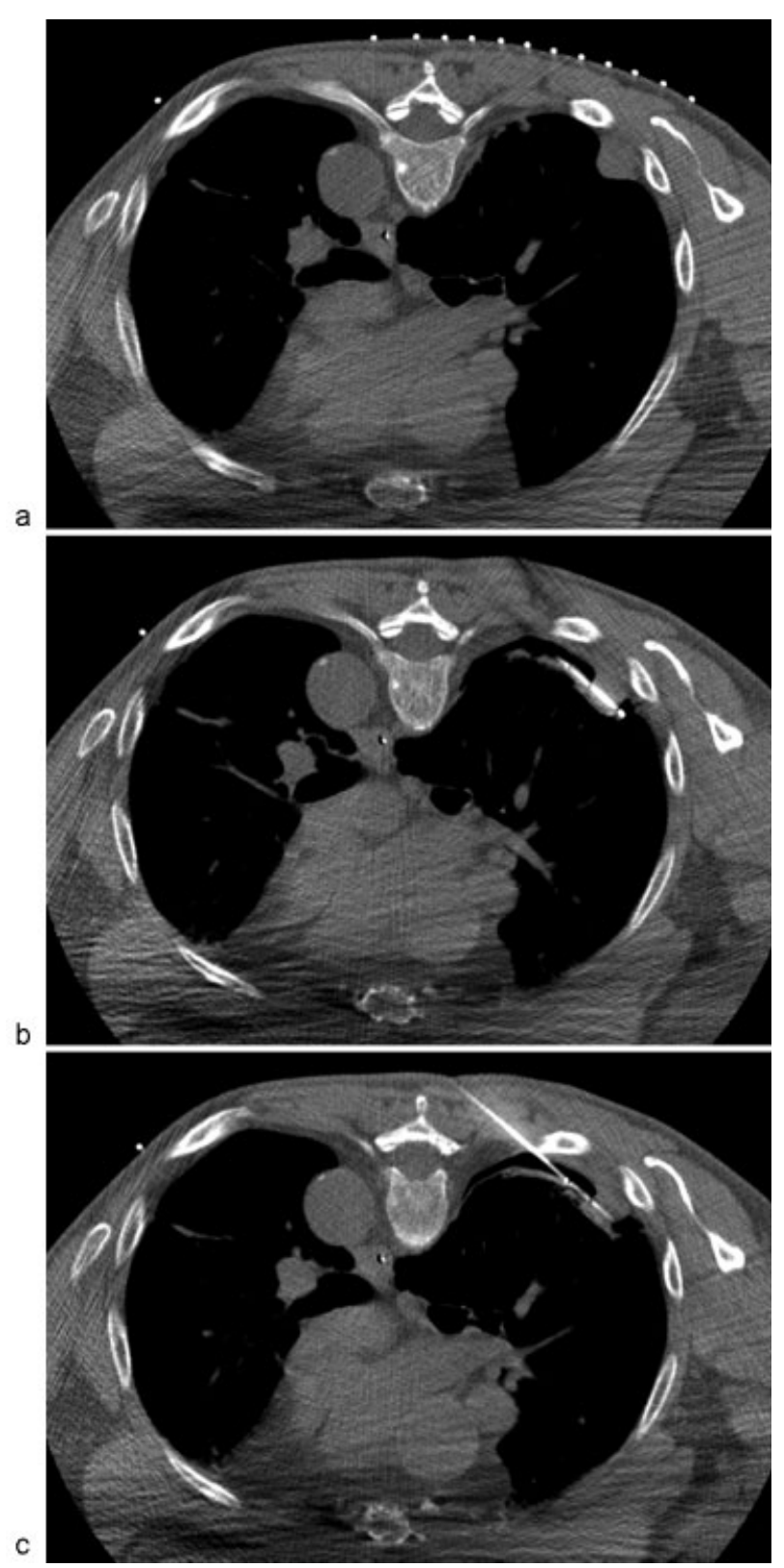

Fig. 3 Cryoablation of a pleural-based tumor in a patient with chest wall pain. Cryoablation was favored over RFA or MWA, as it provides pain palliation and is safer for tumors adjacent to the chest wall. (a) Preprocedural CT image shows pleural-based tumor in the right lower lobe (arrow). (b) Intraprocedural CT image shows the tip of the ablation probe has been advanced into the tumor with intentional creation of a pneumothorax (arrowheads) to protect the chest wall. (c) Intraprocedural CT image shows that the ablation probe trajectory intentionally traverses more lung parenchyma before entering the tumor. This trajectory is preferred over direct puncture into the tumor to limit nontarget injury.

cryoablation devices use 1 to 15 probes that are 1.5 to $2.4 \mathrm{~mm}$ in diameter. ${ }^{32,33}$

Studies have demonstrated the safety and effectiveness of cryoablation in treating pulmonary tumors. In the interim analysis from the ECLIPSE trial of 40 patients with 60 lung metastases ( $40 \%$ colon, $23 \%$ kidney, $8 \%$ sarcoma), the 1 -year local tumor control rate was $94.2 \%$ and the 1 -year overall survival rate was $97.5 \%^{34}$; in this study, the mean size of metastases was $1.4 \mathrm{~cm}$ and the rate of pneumothorax requiring chest tube was $18.8 \%$. In a larger study of 199 metastatic lung tumors and 11 primary lung tumors treated with cryoablation, the local progression-free rates were $80 \%$ at 1 year, $69 \%$ at 2 years, and $68 \%$ at 3 years; local tumor progression was associated with the presence of a vessel at least $3 \mathrm{~mm}$ in diameter within $3 \mathrm{~mm}$ of the tumor. ${ }^{35}$ In a study of medically inoperable patients with NSCLC, cryoablation was effective in the treatment of pulmonary tumors with a 5 -year overall survival of $68 \%$ and a 5 -year progression-free survival of $88 \%$. Major complications rate was $6.4 \%$ including two cases of hemoptysis. ${ }^{36}$

\section{Complications of Ablation}

Lung ablation is generally well tolerated by most patients, even those with limited cardiac or pulmonary reserve. The most common complication after ablation is pneumothorax with a minority of patients requiring chest tube placement. In a study of 3,344 patients who underwent lung ablation (61.9\% primary lung cancers, 38.1\% metastatic lung tumors), most complications were pneumothorax (38.4\%), pneumonia (5.7\%), and pleural effusion (4\%). ${ }^{37}$ Another potential complication is nerve injury depending on the location of the tumor; thermal ablation may cause injury to the brachial plexus or phrenic nerve. ${ }^{38,39}$

Although most complications after ablation are minor, there can be rare but serious complications such as massive pulmonary hemorrhage, bronchopleural fistula, pulmonary artery pseudoaneurysm, air embolism, pneumonitis, and death. In a retrospective single-institution study of 1,000 RFA sessions in 420 patients, the procedure-related mortality was $0.4 \%$ with one death from hemothorax and three deaths from interstitial pneumonia. ${ }^{40}$ Fatal hemoptysis is rare $(<1 \%)$ and has been associated with pulmonary artery pseudoaneurysm ${ }^{41}$ and antiplatelet agent use. ${ }^{42}$ In one series of lung RFA, there were two deaths attributed to interstitial pneumonia; both patients received radiation therapy prior to thermal ablation. ${ }^{43}$

\section{Which Type of Ablation Should I Use?}

Currently, there is no randomized clinical trial comparing RFA, MWA, and cryoablation. There are also no consensus guidelines regarding the optimal selection of ablative technique. In this section, we will only provide our opinions about which ablation device to use for particular clinical scenarios.

Unlike the liver or kidney, the aerated lung has high electric impedance, as air is a thermal insulator. As such, RFA may have limited effectiveness in the lung even when electrodes are placed appropriately in the tumor. ${ }^{44}$ MWA and cryoablation can overcome this inherent limitation in aerated lung. Working at a much higher electromagnetic frequency, MWA creates a more predictable, more confluent, and larger ablation zone that is less susceptible to heat sink effect. ${ }^{45}$ The third generation MWA systems are especially effective in the lung and recommended for use over RFA. 
MWA is also faster than RFA; for example, MWA can sufficiently ablate a lung tumor in only 5 minutes after antenna placement. However, due to its greater power, MWA does have the potential to increase complication rates if not applied appropriately. Optimal antenna positioning is paramount in MWA. Unlike percutaneous needle biopsies, the antenna placement during MWA should usually traverse more (not less) normal lung parenchyma. Some interventionalists recommend $2 \mathrm{~cm}$ of aerated lung between the ablation zone and the pleural surface if possible. For larger peripheral lung tumors, it may be tempting to perform MWA or RFA using the shortest, simplest path. However, when such a "direct" path to the tumor is utilized, the ablation zone may extend unnecessarily into the chest wall and cause prolonged pain, air leak, and skin burn. Therefore, it is advised that the point of entry into lung (i.e., pleural puncture site) excludes the target ablation zone. ${ }^{46}$ Furthermore, tract cautery should not be used in the lung for either RFA or MWA, as it may lead to bronchopleural fistula. At the site of lung ablation, the lung may lose volume due to dehydration so that the ablation probe retracts from the pleural lining resulting in persistent air leak. ${ }^{47}$

From a safety point of view, cryoablation may be advantageous over heat-based devices for the following tumor locations: central/hilar tumors, tumors in or near the mediastinum, and subpleural tumors adjacent to the chest wall. Cryoablation lacks the cautery effect that is inherent with MWA or RFA. Even when the ablation zone extends into the chest wall, cryoablation may be associated with only minimal pain for the patient. For central or mediastinal tumors, cryoablation may be the safer option given the close proximity to nerves, larger blood vessels, and airways. Compared with RFA, cryoablation is also resistant to coldsink effect of ventilation and therefore may be more effective near larger airways. ${ }^{48,49}$ However, the absence of cautery effect in older cryoablation devices does have a potential disadvantage of increased bleeding complications (hemoptysis or hemothorax). Damage to the microcirculation during the thaw cycle of cryoablation may also contribute to the increased bleeding risk. Although pulmonary hemorrhage is usually self-limiting after cryoablation, there have been case reports of uncontrollable hemorrhage leading to death. Lastly, in patients who develop recurrent disease after radiation therapy, ablation should be used with caution, as there is poor wound healing as a result of disorganized vasculature.

\section{Conclusion}

Image-guided ablation is a minimally invasive, safe, repeatable, effective treatment option for patients with primary or metastatic pulmonary tumors. Although the majority of the literature is based on RFA, the use of MWA and cryoablation is increasing due to their efficacy and safety profile. The high-powered MWA appears to offer superior local tumor control, although it must be used properly. To prevent prolonged air leaks and bronchopleural fistulae, tract cautery should not be performed and probe should not be placed directly into the tumor (but rather include normal lung parenchyma). For tumors involving the chest wall or mediastinum, cryoablation is generally favored due to its safety profile. Future trials comparing the ablation modalities may be useful in establishing their relative clinical outcomes.

\section{References}

1 Brambilla E, Travis W. Lung cancer. In: Stewart BW, Wild CP, eds. World Cancer Report. Lyon: World Health Organization; 2014

2 Siegel RL, Miller KD, Jemal A. Cancer statistics, 2016. CA Cancer J Clin 2016;66(01):7-30

3 Kwan SW, Mortell KE, Talenfeld AD, Brunner MC. Thermal ablation matches sublobar resection outcomes in older patients with early-stage non-small cell lung cancer. J Vasc Interv Radiol 2014; 25(01):1-9.e1

4 Kwan SW, Mortell KE, Hippe DS, Brunner MC. An economic analysis of sublobar resection versus thermal ablation for earlystage non-small-cell lung cancer. J Vasc Interv Radiol 2014; 25(10):1558-1564, quiz 1565

5 Binkley MS, Trakul N, Jacobs LR, et al. Colorectal histology is associated with an increased risk of local failure in lung metastases treated with stereotactic ablative radiation therapy. Int J Radiat Oncol Biol Phys 2015;92(05):1044-1052

6 Dupuy DE, Zagoria RJ, Akerley W, Mayo-Smith WW, Kavanagh PV, Safran $\mathrm{H}$. Percutaneous radiofrequency ablation of malignancies in the lung. AJR Am J Roentgenol 2000;174(01):57-59

7 Boston Scientific RF 3000 radiofrequency ablation system. Available at: http://www.bostonscientific.com/templatedata/imports/ collateral/Radiology/broc_rf3000_03_us.pdf. Accessed April 27, 2017

8 StarBurst (RITA) radiofrequency ablation system. Available at: http://www.angiodynamics.com/products/what-is-rfa. Accessed April 27, 2017

9 Cool-Tip Covidien radiofrequency ablation system. Available at: http://surgical.covidien.com/products/ablation-systems/cooltip-rf-ablation-system-e-series. Accessed April 27, 2017

10 Simon CJ, Dupuy DE, DiPetrillo TA, et al. Pulmonary radiofrequency ablation: long-term safety and efficacy in 153 patients. Radiology 2007;243(01):268-275

11 Ambrogi MC, Fanucchi O, Cioni R, et al. Long-term results of radiofrequency ablation treatment of stage I non-small cell lung cancer: a prospective intention-to-treat study. J Thorac Oncol 2011;6(12):2044-2051

12 Chua TC, Sarkar A, Saxena A, Glenn D, Zhao J, Morris DL. Long-term outcome of image-guided percutaneous radiofrequency ablation of lung metastases: an open-labeled prospective trial of 148 patients. Ann Oncol 2010;21(10):2017-2022

13 Palussière J, Marcet B, Descat E, et al. Lung tumors treated with percutaneous radiofrequency ablation: computed tomography imaging follow-up. Cardiovasc Intervent Radiol 2011;34(05): 989-997

14 de Baère T, Aupérin A, Deschamps F, et al. Radiofrequency ablation is a valid treatment option for lung metastases: experience in 566 patients with 1037 metastases. Ann Oncol 2015; 26(05):987-991

15 Dupuy DE, Fernando HC, Hillman S, et al. Radiofrequency ablation of stage IA non-small cell lung cancer in medically inoperable patients: results from the American College of Surgeons Oncology Group Z4033 (Alliance) trial. Cancer 2015;121(19):3491-3498

16 Lencioni R, Crocetti L, Cioni R, et al. Response to radiofrequency ablation of pulmonary tumours: a prospective, intention-totreat, multicentre clinical trial (the RAPTURE study). Lancet Oncol 2008;9(07):621-628 
17 Simon CJ, Dupuy DE, Mayo-Smith WW. Microwave ablation: principles and applications. Radiographics 2005;25(Suppl 1): S69-S83

18 Evident (Covidien) microwave ablation system. Available at: http:// surgical.covidien.com/products/ablation-systems. Accessed April 27, 2017

19 Microtherm X. BSD microwave ablation system. Available at: http://www.bsdmedical.com/usa/brochures/Microthermx_Brochure.pdf. Accessed April 27, 2017

20 Avecure Medwaves microwave ablation system. Available at: http://www.medwaves.com/. Accessed April 27, 2017

21 Certus 140 Neuwave Medical microwave ablation system. Available at: http://www.neuwavemedical.com/NeuWaveMedicalFilePile/ NeuWaveFDAClearancePress20101028Final9.pdf. Accessed April 27, 2017

22 AMICA microwave ablation system. Available at: http://www.amarantoweb.com/hsmedical/drive/File/AMICA_eng.pdf. Accessed April 27, 2017

23 Acculis MTA microwave ablation system. Available at: http:// www.angiodynamics.com/products/Acculis. Accessed April 27, 2017

24 Wolf FJ, Grand DJ, Machan JT, Dipetrillo TA, Mayo-Smith WW, Dupuy DE. Microwave ablation of lung malignancies: effectiveness, CT findings, and safety in 50 patients. Radiology 2008; 247(03):871-879

25 Vogl TJ, Worst TS, Naguib NNN, Ackermann H, Gruber-Rouh T, Nour-Eldin N-EA. Factors influencing local tumor control in patients with neoplastic pulmonary nodules treated with microwave ablation: a risk-factor analysis. AJR Am J Roentgenol 2013; 200(03):665-672

26 Belfiore G, Ronza F, Belfiore MP, et al. Patients' survival in lung malignancies treated by microwave ablation: our experience on 56 patients. Eur J Radiol 2013;82(01):177-181

27 Gage AA, Baust J. Mechanisms of tissue injury in cryosurgery. Cryobiology 1998;37(03):171-186

28 Izumi Y, Oyama T, Ikeda E, Kawamura M, Kobayashi K. The acute effects of transthoracic cryoablation on normal lung evaluated in a porcine model. Ann Thorac Surg 2005;79(01):318-322, discussion 322

29 Hinshaw JL, Littrup PJ, Durick N, et al. Optimizing the protocol for pulmonary cryoablation: a comparison of a dual- and triplefreeze protocol. Cardiovasc Intervent Radiol 2010;33(06): 1180-1185

30 Yamauchi Y, Izumi Y, Hashimoto K, et al. Percutaneous cryoablation for the treatment of medically inoperable stage I non-small cell lung cancer. PLoS One 2012;7(03):e33223

31 Maiwand MO. The role of cryosurgery in palliation of tracheobronchial carcinoma. Eur J Cardiothorac Surg 1999;15(06): 764-768

32 Endocare cryotherapy system. Available at: https://www.healthtronics.com/physicians/cryotherapy/cryotherapy-services-andsolutions. Accessed April 27, 2017

33 Cryohit cryotherapy system. Available at: http://www.galilmedical.com/. Accessed April 27, 2017
34 de Baere T, Tselikas L, Woodrum D, et al. Evaluating cryoablation of metastatic lung tumors in patients-safety and efficacy: the ECLIPSE trial-interim analysis at 1 year. J Thorac Oncol 2015; 10(10):1468-1474

35 Yashiro H, Nakatsuka S, Inoue M, et al. Factors affecting local progression after percutaneous cryoablation of lung tumors. J Vasc Interv Radiol 2013;24(06):813-821

36 Moore W, Talati R, Bhattacharji P, Bilfinger T. Five-year survival after cryoablation of stage I non-small cell lung cancer in medically inoperable patients. J Vasc Interv Radiol 2015;26(03):312-319

37 Welch BT, Brinjikji W, Schmit GD, et al. A national analysis of the complications, cost, and mortality of percutaneous lung ablation. J Vasc Interv Radiol 2015;26(06):787-791

38 Hiraki T, Gobara H, Mimura H, et al. Brachial nerve injury caused by percutaneous radiofrequency ablation of apical lung cancer: a report of four cases. J Vasc Interv Radiol 2010;21(07):1129-1133

39 Matsui Y, Hiraki T, Gobara H, et al. Phrenic nerve injury after radiofrequency ablation of lung tumors: retrospective evaluation of the incidence and risk factors. J Vasc Interv Radiol 2012;23(06): 780-785

40 Kashima M, Yamakado K, Takaki H, et al. Complications after 1000 lung radiofrequency ablation sessions in 420 patients: a single center's experiences. AJR Am J Roentgenol 2011;197(04): W576-80

41 Borghol S, Alberti N, Frulio N, et al. Pulmonary artery pseudoaneurysm after radiofrequency ablation: report of two cases. Int J Hyperthermia 2015;31(01):1-4

42 Vaughn C, Mychaskiw G II, Sewell P. Massive hemorrhage during radiofrequency ablation of a pulmonary neoplasm. Anesth Analg 2002;94(05):1149-1151

43 Nomura M, Yamakado K, Nomoto Y, et al. Complications after lung radiofrequency ablation: risk factors for lung inflammation. $\mathrm{Br}$ J Radiol 2008;81(963):244-249

44 Beland MD, Wasser EJ, Mayo-Smith WW, Dupuy DE. Primary non-small cell lung cancer: review of frequency, location, and time of recurrence after radiofrequency ablation. Radiology 2010; 254(01):301-307

45 Durick NA, Laeseke PF, Broderick LS, et al. Microwave ablation with triaxial antennas tuned for lung: results in an in vivo porcine model. Radiology 2008;247(01):80-87

46 Lee KS, Takaki H, Yarmohammadi H, et al. Pleural puncture that excludes the ablation zone decreases the risk of pneumothorax after percutaneous microwave ablation in porcine lung. J Vasc Interv Radiol 2015;26(07):1052-1058

47 Sakurai J, Hiraki T, Mukai T, et al. Intractable pneumothorax due to bronchopleural fistula after radiofrequency ablation of lung tumors. J Vasc Interv Radiol 2007;18(1, Pt 1):141-145

48 Anai H, Uchida BT, Pavcnik D, et al. Effects of blood flow and/or ventilation restriction on radiofrequency coagulation size in the lung: an experimental study in swine. Cardiovasc Intervent Radiol 2006;29(05):838-845

49 Hinshaw JL, Sampson L, Lee FTJ Jr, Laeseke PF, Brace CL. Does selective intubation increase ablation zone size during pulmonary cryoablation? J Vasc Interv Radiol 2008;19(10):1497-1501 